\title{
The principle of distance during COVID-19 outbreak in Korea
}

\author{
Kyoo-Man $\mathrm{Ha}^{1}$
}

Received: 9 July 2020 / Revised: 9 July 2020 / Accepted: 20 July 2020 / Published online: 27 July 2020

(C) Springer Nature Switzerland AG 2020

\begin{abstract}
The majority of confirmed COVID-19 cases in Korea have been related to mass infection. The nation must urgently promote the distance awareness approach during emergency response and discourage the distance unawareness approach. Other nations may also apply the principle of distance to their pandemic management.
\end{abstract}

Keywords Coronavirus $\cdot$ Distance unawareness $\cdot$ Distance awareness $\cdot$ Mass infection $\cdot$ Public gatherings

\section{Introduction}

The 2019 coronavirus disease (COVID-19) as an epidemic respiratory disease is new to human society; hence, the international community does not yet know how to efficiently prevent or treat it. Instead, various nations have tried different approaches to COVID-19 based on the responses to previous respiratory diseases (Hjelmgaard 2020). Examples include precautions such as wearing face masks, washing hands with soap and running water, sneezing into one's upper sleeve, restricting travel, closing restaurants/bars, maintaining distance among people, testing for coronavirus with testing kits, using ibuprofen, and so on.

South Korea (hereinafter Korea) has been no exception to the impact of the COVID-19 outbreak. The risk of the pandemic has steadily acquired gravity in the current times, with the loss of human lives, economic damages, and psychological impacts being observed. A noticeable factor is that more than $80 \%$ of the confirmed cases have been related to mass infection, while less than $20 \%$ have been linked to individual infection (KCDC 2020). Mass infection refers to a situation where many pathogens enter human tissues or circulatory systems and thus result in breakouts among multitudes. Considering that the Korean environment has been ripe for mass infection, this issue should be solved on a war footing.

Kyoo-Man Ha

ha1999@hotmail.com

1 Department of Emergency Management, Inje University, 197 Inje-ro, Gimhae City, Gyeongnam 50834, South Korea
This paper aims to highlight the importance of maintaining a distance between people during the COVID-19 outbreak in Korea, with the ultimate goal of reducing mass infections. In doing so, the paper has analyzed the distance unawareness approach versus the distance awareness approach. Unawareness is not knowing the distance to be maintained between people (at least $6 \mathrm{ft}$ or $2 \mathrm{~m}$ ), whereas awareness refers to a perception of the aforementioned distance. These two approaches have been compared in terms of four major stakeholders: governments, religious groups, hospitals, and other community-based institutions. The primary argument is that Korea must ensure a transformation from the distance unawareness approach to the distance awareness approach.

\section{Distance unawareness approach}

Governments have provided several guidelines to people on how to efficiently deal with COVID-19. Examples include wearing face masks, washing hands with soap and running water, using hand sanitizers, avoiding contact with people, boiling food, avoiding the use of public toilets due to infection through fecal contamination, combating aerosol infection, and so on. In general, governments have not stressed the critical issue of maintaining a distance among people. As a result, even government employees, such as local officials, soldiers, prison officers, and so on, have been infected.

Several religious groups have not changed their mode of conduct of religious services during the outbreak of COVID19. The persistence of small distances and close body contact among worshippers have turned people into sources of mass infection in Korea. An example is the Shincheonji Church of 
Jesus, a secretive cult that has made many believers pray in a relatively crowded space (Rashid 2020). Each believer keeps a very short distance from another during services and this has resulted in a super-spreading of the infection. Further, the followers of this Church have been extremely uncooperative by refusing infection testing, citing the alleged invasion of their privacy. The Oncheon church, a regular cult, has also produced some infected patients due to close proximity among believers.

Acknowledging that COVID-19 is a contagious virus causing respiratory illnesses, medical staff in hospitals have fully recognized the necessity of maintaining a distance among potential patients. In a short time, a majority of hospitals have appreciated the need for distance amidst the coronavirus outbreak more than other stakeholders. Nonetheless, some hospitals, such as the Cheongdo Daenam hospital, Daegu Munsung hospital, a few sanatoriums, and other nursing homes, have failed to follow this practice. In particular, the Cheongdo Daenam hospital's poor response has led to mass infections on its fifth floor.

Some community-based institutions have also not abided by the principle of distance among their members. Rather, members have tried to wear face masks and wash hands alone to prevent infection. These institutions include Zumba dance clubs, private call centers, Hanmaum apartments, and certain families as well. All of them are responsible for causing mass infection and thus producing a multitude of patients. In a sense, despite the experience of the emergency response to the Middle East Respiratory Syndrome (MERS) in 2015 and the issue of hospital infections, these institutions have not cared much about the issue of distance among members during the current crisis.

\section{Distance awareness approach}

As COVID-19 is a virus new to human society, nobody is sure of its mechanisms yet. It is understandable that governments have made all efforts to issue multiple guidelines on how to respond to COVID-19. However, too many guidelines have caused panic among people and thus confusion in their emergency responses. Therefore, governments need to emphasize authentic guidelines, such as maintaining distances among people, without too much stress on untested measures such as wearing face masks in public.

During the outbreak of COVID-19, it is the need of the hour that religious followers refrain from religious gatherings. Many believers of Shincheonji and other religious groups have been stubborn in the practice of their faiths by conducting religious services in confined spaces, regardless of the outcome. However, considering that the COVID-19 outbreak is a national emergency, all believers must be aware that maintaining a distance among themselves is a preventive measure against the pandemic. They may contribute to disease prevention during emergency response by holding Internet church services.

However, saying is one thing; doing is quite another. When healthcare institutions including Daenam hospital, Munsung hospital, sanatoriums, and nursing homes were aware of the significance of distance between their patients, they should have consistently practiced it from the beginning. As professional healthcare providers, hospital authorities must isolate infected patients from common spaces and prevent them from mingling with others. Patients who are old or those with comorbidities such as diabetes and lung diseases should be put into isolation wards, while about $80 \%$ of the mild cases should be treated through temporary treatment centers (Woodard et al. 2020).

Since the COVID-19 outbreak in China, it has been directly or indirectly known to Korea that the speed of the coronavirus infection spread is relatively high in communities. Hence, to efficiently deal with COVID-19, community-based institutions in Korea should practice maintaining a distance among their members and should not allow members' gatherings or assemblies in limited spaces specifically to avoid mass infection. Rather, each member must stay in their home and use appropriate communication tools instead.

\section{Major discussion}

A few Korean stakeholders have been aware of the importance of maintaining a distance among people during the emergency response to the COVID-19 outbreak. However, this has not been satisfactory enough for the nation, considering that many stakeholders have ignored or forgotten the principle of distance. With that said, the need of the hour is that Korea must move from the distance unawareness approach to the distance awareness approach to the fullest extent, while making other efforts against the COVID outbreak.

To fully eradicate COVID-19, all the four stakeholders should remain aware of the principle of distance. When any stakeholder fails to keep a distance in private/public gatherings or congregation settings, COVID-19 will immediately turn into a super-spreader and thus causes mass infection. Thus, COVID-19 will continue to cause not only physical impacts but also significant social impacts on society. While being counseled to keep a distance, Koreans should refrain from participating in communally irresponsible activities for the time being.

It would be best for individuals to not leave their homes during the COVID-19 outbreak. However, this action is only theoretically possible. As a practical alternative, all individuals must religiously keep a distance from others during their daily lives. In doing so, all individuals have to practice social distancing, including avoiding handshakes, undertaking shift 
work, opting for telework, closing buildings as well as avoiding mass gatherings (Ahmed et al. 2018). In extreme cases, they need to implement isolation, quarantine, lockdown, and so on.

The Korean experience of dealing with the COVID-19 outbreak may produce a good learning experience for others. Nations that have not faced the full extent of the coronavirus threat yet may enforce the golden rule of social distance from the outset. Other nations that are currently dealing with the crisis can further address the issue of maintaining a distance among people. If they fail to do so, COVID-19 will spread all over the world and thus become a formidable threat. Besides, mutations of the coronavirus in other countries can spread to Korea and thereby cause far greater impacts in local areas.

\section{Compliance with ethical standards}

Conflict of interest The author declares that he has no conflict of interest.

\section{References}

Ahmed F, Zviedrite N, Uzicanin A (2018) Effectiveness of workplace social distancing measures in reducing influenza transmission: A systematic review. BMC Pub Health 18:518

Hjelmgaard K (2020) These countries are doing the best and worst jobs fighting coronavirus. USA Today. https://www.usatoday.com/story/ news/world/2020/03/17/coronavirus-how-countries-across-globeresponding-covid-19/5065867002/. Accessed 9 Jul 2020

Korea Centers for Disease Control and Prevention (KCDC) (2020) Official Website of KCDC (in Korean). https://www.cdc.go.kr/ CDC/main.jsp. Accessed 7 Jul 2020

Rashid R (2020) Being called a cult is one thing, being blamed for an epidemic is quite another. The New York Times https://www. nytimes.com/2020/03/09/opinion/coronavirus-south-korea-church. html. Accessed 4 Jul 2020

Woodard A, Su R, Gal S (2020) What to know about the coronavirus outbreak in 9 chars and maps. Business Insider. https://www. businessinsider.com/coronavirus-in-charts-covid-19-symptomsspread-deaths-warnings-2020-2. Accessed 27 Jul 2020

Publisher's note Springer Nature remains neutral with regard to jurisdictional claims in published maps and institutional affiliations. 